Orthotics

\section{Plagiocephaly and head binding}

\section{S J Bridges, T L Chambers, I K Pople}

\section{Orthotic devices do not improve plagiocephaly}

T he practice of head deformation by pressure to an infant's skull dates back to 2000 BC when the Ancient Egyptians used head binding to produce a cosmetically pleasing and fashionable skull shape. ${ }^{1}$ With an increasing incidence of plagiocephaly (asymmetric skull) this practice, with a modern slant, is re-emerging. A simple web search resulted in five "paediatric offices" offering such a service. If an Ancient Egyptian walked into clinic today with their child's head bound between two planks of wood, we would be informing social services. Should we, as paediatricians, be advocating modern orthotic devices for plagiocephaly or condemning them?

Plagiocephaly can be subdivided into synostotic, where one or more sutures are fused, and nonsynostotic, or deformational, plagiocephaly. Surgical treatment of the synostotic variety is undisputed as the deformity is likely to progress and there is a significant risk of raised intracranial pressure. However the treatment of deformational plagiocephaly is more controversial.

There are no population based studies to establish the precise incidence or prevalence of deformational plagiocephaly, but the number of referrals to both paediatric and surgical units is increasing. ${ }^{2-4}$

Posterior deformational plagiocephaly occurs more commonly on the right and there is a notable male predominance. The laterality may be in part a result of intrauterine position with $85 \%$ of vertex presentations lying on the left occipital anterior position. If the baby descends into the pelvis (fig 1 ), this may limit the growth of the right occiput and left frontal areas. $^{56}$ The asymmetry may be further exacerbated postnatally-when the child is laid supine, the head will automatically roll to the flattened side, which then becomes the preferred side for sleeping.

This hypothesis also explains the increase in incidence of posterior deformational plagiocephaly since the "Back to Sleep" recommendations for prevention of sudden infant death syndrome. ${ }^{2}{ }^{3}$ Mulliken et al showed that over a four year period from 1992 to 1996, the incidence of frontal plagiocephaly (attributed to prone sleeping) decreased and almost disappeared, with a concomitant rise in posterior plagiocephaly. ${ }^{7}$
Suggested explanations for the male predominance have been that the male head is larger and grows more rapidly than that of the female, ${ }^{7}$ and that male fetuses are less flexible than their female counterparts. ${ }^{8}$ Both these factors result in the male infant being more at risk of developing deformational anomalies.

Deformational plagiocephaly has not been shown to be associated with any long term problems, although a concern regarding strabismus has been raised by some authors as a result of traction on the ocular globe from the underlying deformed bone in frontal plagiocephaly. ${ }^{9}$ However, this has only been described in synostotic plagiocephaly and there is no evidence to support this theory in deformational plagiocephaly. Further reassurance on this point is provided by the fact that the majority of plagiocephaly cases are currently posterior in nature as described above. ${ }^{7}$ As the head enlarges the relative asymmetry reduces and hair growth disguises any minor anomalies. In view of the lack of complications and the potential for natural resolution, treatment is entirely cosmetic and should therefore confer minimal risk or discomfort to the patient.

Suggested treatments include simple reassurance, positioning and physiotherapy, external orthotic devices, or

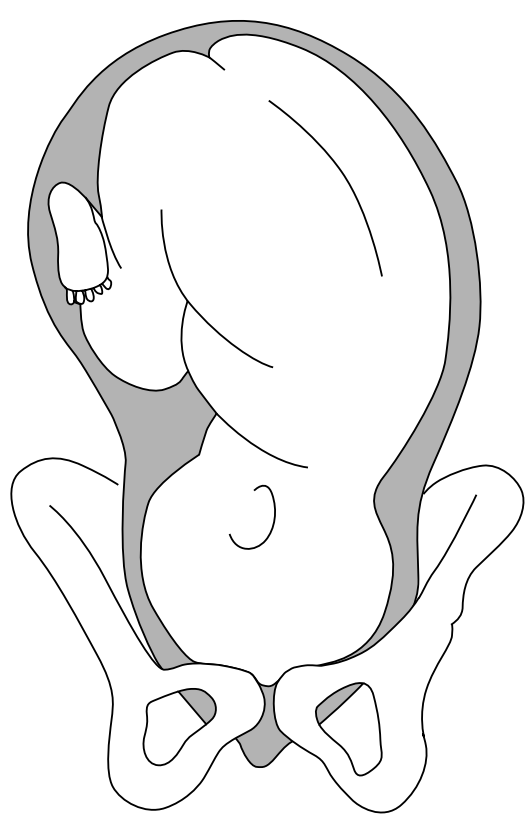

Figure 1 Left occipital anterior position. finally surgery. Reassurance and explanation of the usual lack of long term problems may be sufficient for the milder cases. However, for parents with real concerns about cosmetic appearance, what more can be done? Surgery does confer a risk, albeit minimal, in view of the close proximity of the posterior dural venous sinuses, ${ }^{10}$ and in view of this, is unsuitable for the majority of cases. This leaves either orthoses or positioning and physiotherapy as alternatives. Unfortunately evidence from the literature for either of these interventions is limited. There have been no randomised trials and the best available evidence is contained within prospective cohort studies.

"Positioning" infants refers to advising parents to lay the child's head on the opposite side to that which is flattened when lying down. This can be facilitated by placing objects of interest on that side of the cot to encourage head movement in that direction. Some authors advocate assisting positioning with foam wedges to ensure that the head is held in the required orientation. Physiotherapy is particularly useful if there is any sternocleidomastoid imbalance, with or without evidence of torticollis. This has been identified in $25-75 \%$ of children with plagiocephaly. ${ }^{11}{ }^{12}$ Whether this is a cause or effect of the plagiocephaly is uncertain.

Positioning and physiotherapy is thought to be more effective if it is instituted before 6 months of age; beyond that age the child is normally fairly mobile and more likely to change position during sleep independently.

$\mathrm{O}^{\prime}$ Broin and colleagues ${ }^{12}$ and Pople and colleagues ${ }^{13}$ have both studied the natural history of children referred with deformational plagiocephaly and managed with sleep positioning and physiotherapy alone. Both authors used a qualitative scale based on physician and parental views of the asymmetry. All patients were thought to show a significant improvement in asymmetry at follow up over 6-12 months, although the asymmetry did not resolve completely in all patients. These studies could be criticised for their non-objective qualitative measurements, but these views may be more clinically relevant than actual measurements when it comes to acceptability of cosmetic results.

External orthoses include both dynamic and passive devices such as skull bands or helmets. These devices either compress the prominent part of the skull or only allow growth in the flattened part of the skull. A specialised technician is required, as each device is custom made for that particular child. The orthoses are in place for 15-22 hours each day and require regular review to ensure skin viability and circumferential growth of the head. Orthoses are only 
thought to be beneficial in the majority of cases if the child is aged less than 12 months, as $85 \%$ of postnatal head growth occurs in the first year of life. ${ }^{14}$ There has been a concern from some centres regarding stigmatisation of helmet wearers; however, this is not a factor that has been recognised locally.

A range of clinical studies ${ }^{15-18}$ have charted the change in anthropometric measurements with orthotic treatment. They show what would appear to be an impressive asymmetrical resolution with a mean change in asymmetry of about 5 $\mathrm{mm}$. The main problem with each of these studies is the lack of controls. Without a set of control data there is no way of knowing whether the intervention is actually beneficial.

There is only one study that compares the outcome in more than one treatment group. Mullikan and colleagues ${ }^{7}$ provide control data on patients that chose not to have the orthotic intervention-in this case a helmet. Despite its failings this study does provide the best evidence and appears to show a significant reduction of asymmetry $(p<0.001)$ in the treatment group. However, the lack of randomisation and obvious physician bias, with two of three parents opting for the helmet, makes interpretation of the results difficult.

There does not appear to be any definite evidence for improved outcome with orthoses-so are there any disadvantages? The factors that immediately spring to mind are cost and inconvenience. In the USA it has been estimated that the cost of an external cranial orthosis treatment is equivalent to a minor neurosurgical procedure such as ventriculoperitoneal shunt revision. ${ }^{19}$ Some health insurance companies have denied authorisation for such treatment. Concern has also been raised regarding whether orthoses may restrict cranial growth. There is no evidence to support this, but is one of the reasons why children are followed up weekly to ensure that no complications have occurred. ${ }^{15} 16$ Whether orthoses cause any discomfort to these babies can only be guessed at and probably differs on an individual basis. Should we be risking a child's discomfort, family inconvenience, and NHS funding on a treatment that has no clear benefit?

In summary, there is no clear evidence as yet that orthotic devices improve plagiocephaly in the long term. Craniosynostoses should be excluded by clinical and radiological means, before advice is given regarding sleep position and physiotherapy. Neurosurgical or plastic surgery referrals should be reserved for progressive or severe cases. Population based studies are required; these should investigate the natural history of plagiocephaly with qualitative and quantitative measurements. Unless it is found that children have a detrimental outcome as a result of conservatively managed plagiocephaly, modern head binding cannot be recommended as a routine treatment. Indeed, as is the case with many treatments for cosmetic deformity, category l evidence for efficacy of any intervention is lacking and it is often the parents or consumers who drive the treatment trends. If the infant were asked, he might opt for wait and see.

Arch Dis Child 2002;86:144-145

\section{Authors' affiliations}

S J Bridges, T L Chambers, I K Pople, Neonatal SpR, Southmead Hospital, Bristo $\mathrm{BS} 10$ 5NB, UK

Correspondence to: Dr S J Bridges, Neonatal SpR, Southmead Hospital, Bristol BS 10 5NB, UK; sarahbridges@hotmail.com

\section{REFERENCES}

BDSM-online.com. Alternative sexuality and lifestyle resource.

2 Argenta LC, David LR, Wilson JA, Bell WO An increase in cranial deformity with supine sleeping position. J Craniofac Surg 1996;7:5-11.
3 Turk AE, McCarthy JG, Thorne CCHM Wisoff JH. The back to sleep campaign and deformational plagiocephaly: is there a cause for concern? J Craniofac Surg 1996;7:12-18.

4 Kane A, Mithchell L, Craven K, Marsh J.

Observations on a recent increase in plagioceohaly without synostosis. Pediatrics 1996;97:877-85.

5 Hansen M, Mulliken JB. Frontal plagiocephaly: diagnosis and treatment. Clin Plast Surg 1994:21:543-53.

6 Bruneteau RJ, Mulliken JB. Frontal plagiocephaly: synostotic, compensational, or deformational. Plast Reconstr Surg 1992;89:21-31.

7 Mulliken JB, Vander Woude JL, Hansen M et al. Analysis of posterior plagiocephaly: deformational versus synostotic. Plast Reconstr Surg 1999;103:371-80.

8 Graham J Jr. Hip dislocation. In: Smiths's recognizable patterns of human deformation. Philadelphia: Saunders, 1998:P22.

9 Fredrick DR, Mulliken JB, Robb RM. Ocular manifestations of deformational plagiocephaly. J Pediatr Opthalmol Strabismus 1993;93:92-5.

10 Jones BM, Hayward R, Evans R, Britto J. Occipital plagiocephaly; an epidemic of craniosynostosis. BM 1997;315:693-4.

11 Golden KA, Beals SP, Littlefield TR, Pomatto JK. Sternocleidomastoid imbalance versus congenital muscular torticollis: their relationship to positional plagiocephaly. Cleft Palate Craniofac J 1999;36:256-61.

12 O'Broin ES, Allcutt D, Earley M. Posterior plagiocephaly: proactive conservative management. Br J Plast Surg 1999;52:18-23.

13 Pople IK, Sanford RA, Muhlbauer MS Clinical presentation and management of 100 infants with occipital plagiocephaly. Pediatr Neurosurg 1996;25:1-6.

14 Pomerance HH. Growth standards in children. Hagerstown, MD: Harper and Row, 1979.

15 Kelly KM, Littlefield TR, Pomatto JK, et al. Importance of early recognition and treatmen of deformational plagiocephaly with orthotic cranioplasty. Cleft Palate Craniofac 1999-36:127-30.

16 Pollack IF, Losken HW, Fasick P. Diagnosis and management of posterior plagiocephaly. Pediatrics 1997;99:180-5.

17 Roddi R, Jansen MA, Vaandrager JM, van der Meulen JC. Plagiocephaly-new classification and clinical study of 100 patients. J Craniomaxillofac Surg 1995;23:347-54.

18 Ripley CE, Pomatto J, Beals SP, et al. Treatment of positional plagiocephaly with dynamic orthotic cranioplasty. J Craniofac Surg 1994;5:150-9

19 Moss SD. Nonsurgical, nonorthotic treatment of occipital plagiocephaly: what is the natura history of the misshapen head? I Neurosurg 1997;87:667-70.

\section{COMMENTARY}

T he debates related to true synostotic plagiocephaly (unicoronal synostosis or rarely lambdoid synostosis) as opposed to deformational plagiocephaly have raged over the last decade. A number of articles highlight the clinical features that distinguish these conditions. ${ }^{1-3}$ Various terms are applied to non-synostotic calvarial asymmetry, including "deformational plagiocephaly". Plagiocephaly without synostosis (PWS) is the preferred term in our unit. It is generally accepted that plagi- ocephaly without any evidence of synostosis usually needs no surgical intervention.

The exact association between PWS and the "Back to Sleep" campaign is difficult to quantify. A number of obstetrics factors appear to predispose to a child being born with an initially asymmetric head, including multiple pregnancy, fetal malposition or malrotation, and prematurity.

In addition, in our unit we have documented a 54\% incidence of wormian bones in PWS (versus a 17\% incidence in age matched controls). This raises the potential contributing factor of a slightly more malleable head. Interestingly
Mayan headbinding cultures have been shown on archaeological skull findings to have increased numbers of wormian bones. ${ }^{4}$ Whether this represents evidence of increased malleability or whether the reponse to an initial restrictive force is a compensatory division of the sutures is unfortunately impossible to prove.

Important in PWS is the fact that whatever the degree of asymmetry present at birth, flattening frequently progresses over approximately the first six months as pressure is maintained on the back of the head due to the sleeping posture (even more significantly in children with developmental delay and poor tone). The unwary may class this initial 
progression as evidence of a synostic process.

Bridges et al have indicated the difficulty of scientifically assessing outcomes and producing statistical significance, as opposed to assessing clinically relevant effectiveness. The following points need to be taken into account.

(1) There is a form of susceptibility which makes some children flatten more significantly than others; thus not all children nursed on their backs will develop occipital flattening.

(2) Initial early progression usually precedes resolution.

(3) In the vast majority of mild cases, resolution to the point of deformation not being detected other than by someone specifically looking for asymmetry is the "norm".

(4) In more severe cases resolution is slower and may be incomplete, but in the majority of cases proceeds without active intervention to a point where the end result is entirely socially acceptable.

(5) Literature evidence and personal discussion with groups using orthotic devices indicate that their use may slightly speed up the resolution phase but does not conclusively improve the results over patients treated by observation alone.

(6) No studies currently available comment on anything other than the visible "architectural changes". Significant questions need to be raised as to the overall psychosocial effects of placing a child in a helmet unless this is absolutely essential. We believe the interaction between parents and child, and more particularly between the child and non-family members, will be changed by the presence of a helmet, which is frequently viewed by the uninformed as a protective device essentially for a child at risk.

(7) The discomfort of wearing a helmet, particularly in hot weather, and risks associated with general anaesthetic frequently required for adequate fitting of a device, are under emphasised.

It is certainly our feeling that simple observation and allowing nature to take its course is the best option in the vast majority of cases. The authors of the article should be congratulated on their honest reporting of their local experience of these devices and I would definitely support the conclusions drawn in Bridges et al's final paragraph.

S A Wall

Oxford Craniofacial Unit, Radcliffe Infirmary, Oxford OX2 6HE, UK

\section{REFERENCES}

1 Huang MH, Gruss JS, Clarren SK, et al. The differential diagnosis of posterior plagiocephaly: true lambdoid synostosis versus postional molding. Plast Reconstr Surg 1996:98:765-74.

2 Bruneteau RJ, Mulliken JB. Frontal plagiocephaly: synostotic, compensational, or deformational. Plast Reconstr Surg 1992;89:21-31

3 Wall SA. Diagnostic features of the major non-syndromic craniosynostoses and the common deformational conditions which may be confused with them. Current Paediatrics 1997;7:8-17.

4 White $\mathrm{CN}$. Sutural effects of fronto-occipital cranio modifications. Am J Phys Anthropol 1996; 100:347-410.

5 El Jaffer M, Dawson GL. The effects of artificial cranial deformation on the incidence of wormian bones in the lambdoidal sutures. Am J Phys Anthropol 1997:46:155-60.

\section{ADC online submission and review system}

The Editors of $A D C$ are pleased to inform authors and reviewers of its new online submission and review system. Developed by Highwire Press (CA, USA), Bench $>$ Press is a fully integrated electronic system which uses the internet to allow rapid and efficient submission of manuscripts, and for the peer review process to be conducted entirely online. We are the first journal of the BM publishing group to go online in this way; the aim, apart from saving trees, is to speed up the frequently frustrating progress from submission to publication.

Authors can submit their manuscript in any standard word processing software. Standard graphic formats acceptable include: .jpg, .tiff, .gif, eps, etc. The text and graphic files are automatically converted to PDF for ease of distribution and reviewing purposes. Authors are asked to approve their submission before it formally enters the reviewing process. On approval, the submission is passed to the editor and/or reviewers via the web. All transactions are secure. To access the system click on "SUBMIT YOUR MANUSCRIPT HERE" on the ADC homepage: http://www.archdischild.com, or you can access the submission site directly at http://submitadc.bmijournals.com.

We are very excited with this new development and would encourage authors and reviewers to use the system where possible. It is simple to use and should greatly improve on the current peer review process. Full instructions can be found on Bench>Press and ADC online. 